Revista Electrónica Complutense de Investigación en Educación Musical ISSNe: 1698-7454

https://dx.doi.org/10.5209/reciem.63309

\title{
Principios del proceso de enseñanza-aprendizaje de la guitarra flamenca fundamentada en su transmisión oral
}

\author{
Manuel Ángel Calahorro Arjona ${ }^{1}$
}

Recibido: 20 de febrero de 2019 / Aceptado: 11 de junio de 2019

Resumen. La música flamenca; con una clara reminiscencia popular producto del influjo de diferentes pueblos y culturas que han estado en contacto con Andalucía; presenta una serie de características musicales de tal singularidad que le proporcionan un sello de identidad inconfundible. Por el carácter popular que la caracteriza; la transmisión oral siempre ha sido en ella un rasgo destacado. Esa transmisión oral se traduce en toda una metodología tradicional y una serie de procedimientos sobre los que se han fundamentado el proceso de enseñanza y aprendizaje de este arte; cuestión en torno a la cual trataremos en este artículo. Mediante el testimonio oral de artistas que gozan de un notable prestigio en el mundo flamenco actual; establecemos un conjunto de procedimientos que forman parte de esa metodología tradicional y que deberán ir anejos a la enseñanza de este instrumento; ya sea dentro o fuera de un contexto legislado como el conservatorio.

Palabras clave: Guitarra flamenca; transmisión oral; metodología; conservatorio; enseñanza tradicional.

\section{[en] Principles of the teaching-learning process of the flamenco guitar based on its oral transmission}

\begin{abstract}
Flamenco music, with a clear popular reminiscence product of the influence of different peoples and cultures that have been in contact with Andalusia, presents a series of musical characteristics of such uniqueness that provide an unmistakable stamp of identity. Because of the popular character that characterizes it, oral transmission has always been a prominent feature in it. This oral transmission is translated into a whole traditional methodology and a series of procedures on which the teaching and learning process of this art has been based, an issue around which we will deal with in this article. Through the oral testimony of artists who enjoy a remarkable prestige in the current flamenco world, we establish a set of procedures that are part of that traditional methodology and that should be attached to the teaching of this instrument, either inside or outside of a context legislated as the conservatory.
\end{abstract}

Keywords: Flamenco guitar; oral transmission; methodology; conservatory; traditional teaching.

Sumario. 1. Introducción. 2. Método. 3. Resultados: procedimientos anejos a la transmisión oral de la guitarra flamenca. 4. Conclusiones. 5. Referencias bibliográficas.

Cómo citar: Calahorro, M.A. (2019). Principios del proceso de enseñanza-aprendizaje de la guitarra flamenca fundamentada en su transmisión oral. Revista Electrónica Complutense de Investigación en Educación Musical, 16, 117-130.

\footnotetext{
1 Conservatorio Superior de Música "Rafael Orozco" Córdoba

E-mail: manuelflc@hotmail.com
} 


\section{Introducción}

Desde la LOGSE (núm. 283 de 4 de octubre de 1990) y, posteriormente, la LOE (núm. 106 de 4 de mayo de 2006), así como a través de las correspondientes concreciones autonómicas, la especialidad de guitarra flamenca queda incluida dentro de las enseñanzas regladas de conservatorio. Este hecho no solo supuso un paso adelante en la dignificación y valoración de este arte, sino que además permitió el acceso de los músicos flamencos al conocimiento y dominio del lenguaje universal de la música, haciendo uso de la partitura como medio de fijación del material musical y posibilitando el trabajo y análisis de nuevos campos de desarrollo para la guitarra flamenca que antes solo se conocían por intuición como la armonía, melodía, esquemas formales, ritmos o compases.

Ahora bien, el englobar la guitarra flamenca dentro del conjunto de especialidades instrumentales impartidas en los conservatorios, podría acabar derivándose en una equiparación de la metodología con la que se sigue en la enseñanza-aprendizaje de otros instrumentos cuya función principal no es la del acompañamiento al cante, es decir, por un terreno ajeno al tradicional. Como ya señalaron algunos autores (Cera, 2006; Pacheco, 2003), esto podría conllevar el riesgo de que se acabara por implantar una metodología de enseñanza "mal orientada", que olvidara las características propias de la guitarra de acompañamiento, centrándose en exclusiva en la guitarra de concierto. Ya se argumentó en un anterior trabajo (Calahorro, 2019), que en los planes de estudios para las enseñanzas básicas y profesionales de conservatorio hay una deficitaria presencia de las asignaturas de acompañamiento al cante. Esto iría en contra de la propia naturaleza expresiva de la guitarra flamenca, cuya raíz y nacencia, ligada íntimamente al acompañamiento del cante y del baile, está suficientemente atestiguada y documentada (Amat, 1596; Cervantes, 1613; Briceño, 1626; Vargas y Guzmán, 1773; Dembowski, 1841; Ford, 1845; Estébanez, 1847; Jorge, 1860; Peñalba, 1877; Marín, 1902; Delgado, 1906; Grecos, 1973; Batista, 1985; Cano, 1986; Torres, 2009, 2011; Calahorro, 2017, 2018).

Partiendo de la concepción del proceso de enseñanza como un conjunto de acciones formalizadas que se encaminan a provocar el aprendizaje dentro de contextos escolarizados (Bolívar, 1995), queremos razonar que en el caso de la guitarra flamenca este proceso ha de ser matizado, puesto que tradicionalmente se ha aprendido en ámbitos diferentes a los académicos, utilizando unos mecanismos de transmisión que priman la oralidad por encima de la escritura. $Y$ en sus inicios, esta guitarra ha estado marcada por un ritmo espontáneo y dependiente del alma de los artistas, ajeno a un conocimiento musical reglado (Cano, 1986).

Desde antaño, en la enseñanza de la guitarra flamenca, la transmisión oral ha sido el principal medio de traspaso de conocimientos. En este sentido cobran pleno significado las palabras de Bouvier, Bremondy, Joutard, Mathieu y Pelen (1980), convencidos de que en cualquier sociedad alfabetizada, lo esencial de la cultura se transmite de forma oral.

Entendiendo aquí la transmisión oral como el conjunto de enseñanzas, conocimientos y experiencias que se transmiten vivencialmente de maestro a alumno, sin apoyo de la transcripción escrita, en la guitarra flamenca, dichas acciones se han sustentado en buena parte sobre una tradición ágrafa, basándose en un aprendizaje por imitación establecido entre el profesor y el alumno (Pérez, 2005). Si a esto se añade la importancia capital de la propia experiencia y formación que se deriva 
del acompañamiento al cante y al baile, estamos plenamente convencidos que sin esta proyección del aprendizaje por imitación que permita adquirir el repertorio imprescindible para la práctica del acompañamiento al cante y al baile, no se produce un sólido y cabal proceso de aprendizaje en el candidato a músico guitarrista de flamenco.

Autores como Ong (2006), que han estudiado profundamente la fuerza de la oralidad, nos recuerdan que, a diferencia de un contexto alfabetizado, ésta favorece personalidades más comunicativas y menos introspectivas. Y es que la vertiente comunicativa es especialmente necesaria en el desarrollo musical del flamenco, pues gran parte de la interpretación es susceptible de modificación en el mismo momento de la puesta en escena (Calahorro, 2018).

\section{Método}

La heurística, concebida como la búsqueda e investigación de fuentes históricas, ha sido fundamental en la recomposición de un tipo de metodología tradicional de la que se ha valido el flamenco desde antaño. Recurrimos a las fuentes orales tanto como a las escritas, contando con que ambas, en palabras de Joutard "se sostienen mutuamente y se refieren una a la otra" (1983, p. 275). Para la reconstrucción del proceso de enseñanza-aprendizaje en la guitarra flamenca, hemos tenido presente que el flamenco es una música con gran auge y popularidad en la actualidad, por tanto es evidente que hay que recurrir a la documentación escrita, aunque no serán en este caso las únicas fuentes consultadas, pues si así fuera podríamos caer en enfoques archivísticos que desprecian o ignoran otras fuentes de información igualmente interesantes cuando el objeto de estudio así lo demanda.

Para mostrar que los procedimientos y mecanismos en que se sustenta esta transmisión oral es una constante presente ya desde los precedentes musicales de la guitarra flamenca (Torres, 2009), hemos revisado y analizado un elenco de tratados y métodos de guitarra que cuentan con más de cuatro siglos de tradición ininterrumpida (Calahorro, 2018). El punto de partida lo fijamos en el tratado Guitarra española de cinco órdenes, de Joan Carles Amat (1596), escrito para la práctica del estilo rasgueado. Desde entonces, la técnica del rasgueado ha subsistido constante en los ámbitos populares hasta la actualidad, teniendo como principal función la del acompañamiento vocal y de danzas, o en la terminología flamenca, al cante y al baile.

Si la diacronía ha venido dada por esta investigación documental, el testimonio directo de artistas de gran prestigio en el mundo del flamenco actual conforma el estudio sincrónico de dicho trabajo, permitiéndonos individuar una serie de prácticas tan recurrentes como necesarias para el aprendizaje de la guitarra flamenca. Gran parte de estas prácticas estaban ya presentes en muchos de los tratados y métodos analizados, existiendo, por tanto, una plena conexión entre fuentes orales y escritas. Los testimonios, recogidos mediante entrevistas orales semidirigidas, suponían una fuente de información primaria de gran valor, a través de los cuales llegamos a individuar los aspectos que consideraban más importantes en el proceso de enseñanzaaprendizaje de esta disciplina, permitiéndonos hacernos eco también del estado actual de la enseñanza de la guitarra flamenca. 


\subsection{Trabajo de campo: entrevistas}

Desde un primer momento se vio la idoneidad de realizar un trabajo de campo en el que el testimonio oral de artistas de gran prestigio en el arte flamenco diera solidez a nuestro estudio. Siguiendo a Winchester (1996), las entrevistas de tipo cualitativo han de caracterizarse por:

- Muestra reducida: en comparación con un cuestionario cuantitativo, la muestra en una entrevista cualitativa es mucho menos numerosa. En nuestro caso, hemos acotado la muestra a trece entrevistados. Teniendo en cuenta que acudimos tanto a fuentes escritas como a orales y que ambas se sostienen y refieren la una a la otra, nuestras fuentes escritas dan solidez al testimonio de los entrevistados. Por esto, consideramos que el número de entrevistados es lo suficientemente representativo y significativo como para permitir hacer una recomposición clara y real de cuáles son los principios del método tradicional de enseñanza en la guitarra flamenca.

- Elección del personal a entrevistar: la selección del personal entrevistado se ha hecho de forma concienzuda. Aunque cada entrevista buscaba indagar y extraer connotaciones implícitas y explícitas de ese método tradicional de enseñanza, hemos tratado de hacerlas más significativas gracias a la heterogeneidad del personal seleccionado. Esa heterogeneidad ha venido dada por la edad, la formación, el conocimiento del lenguaje musical y la faceta que desarrolla en el flamenco (guitarrista, guitarrista/profesor, cantaor, bailaor o productor).

- Entrevistas extensas: nuestra herramienta ha sido la entrevista oral semidirigida, mediante la cual, aunque se predefinen una serie de cuestiones-guía básicas que guían la conversación, se deja abierta la posibilidad de que a lo largo de la entrevista salgan a la luz nuevas cuestiones que sean relevantes en relación con el objetivo del trabajo y que puedan ampliarlo.

A través de esta técnica llegamos a individuar el estado actual de la enseñanza de la guitarra flamenca y hemos indagado en las experiencias y vivencias más directas y personales de los artistas flamencos - particularmente de la guitarra-, para extraer los aspectos que consideran más importantes en el proceso de enseñanza-aprendizaje de esta disciplina.

Hemos tenido muy presente algunas premisas básicas propuestas por Corbetta (2003) en la realización de las entrevistas, que sintetizamos a continuación: favorecer una explicación previa al entrevistado para evitar posibles inseguridades o desconfianzas; diferenciación entre las preguntas primarias y secundarias, que indagan sobre las primeras; realización de sucintas síntesis de aquello que el entrevistador considera más relevante, buscando la aprobación o ampliación por parte del entrevistado; estímulos de interés a lo largo del discurso que denotan un interés por nuestra parte; pausas que permiten la reflexión del entrevistado; y, finalmente, la utilización de un lenguaje claro y accesible por parte del entrevistado.

Aunque cada uno de estos encuentros se ha desarrollado muy diferentemente y han aflorado cuestiones muy diversas y personales, exponemos a continuación las preguntas formuladas que fueron comunes a todos los entrevistados y que al mismo tiempo nos posibilitaron ir encadenando y sucediendo los diferentes campos temáticos: 
- ¿Qué metodología emplearon tus maestros o profesores en tu formación como músico flamenco?

- En la enseñanza de esta disciplina, ¿qué metodología es la que utilizas?

- ¿Consideras el flamenco una música de transmisión oral?

- ¿Qué importancia otorgas al acompañamiento del cante y del baile en la formación del guitarrista?

- ¿Qué importancia das al estudio de la guitarra de concierto?

\subsection{Muestra}

En la búsqueda y selección de este personal hemos tenido muy presente que nuestros "informantes" o entrevistados tuvieran un prestigio reconocido en el mundo del flamenco. Al ser éste un trabajo de campo circunscrito a la elaboración de una tesis doctoral que se ha ido retroalimentando con las fuentes bibliográficas que se han ido analizando, la extensión temporal en que se sucedieron los encuentros abarca desde el 2011 hasta el 2017. Todas las entrevistas fueron grabadas y transcritas, incorporándose en los anexos del citado trabajo. Sintetizamos a continuación el perfil de cada entrevistado:

- Paco Serrano: profesor del conservatorio superior de Córdoba y guitarrista flamenco de reconocido prestigio tanto en la guitarra de concierto como en la de acompañamiento al cante y al baile. Entrevistado el 11 de noviembre de 2011 en Córdoba.

- Pepe Piñana: profesor del conservatorio de danza de Murcia y guitarrista flamenco de acompañamiento al cante y al baile. Entrevistado el 22 de diciembre de 2011 en Murcia.

- Manolo Franco: profesor del conservatorio superior de Córdoba y guitarrista flamenco de reconocido prestigio en su labor de acompañante al cante, destacado también por su recorrido artístico como concertista. Entrevistado el 4 de enero de 2012 en Córdoba.

- Juan Manuel Cañizares: profesor de la ESMUC de Cataluña y prestigioso concertista de guitarra flamenca. Entrevista escrita recibida el 13 de enero de 2012.

- Paco Cepero: guitarrista flamenco de reconocido prestigio que ha destacado tanto en la guitarra de concierto como en la guitarra de acompañamiento. Su labor compositiva no solo se ha ceñido a la guitarra flamenca, sino que ha sobresalido en la composición de obras vocales para Rocío Jurado, Julio Iglesias o Isabel Pantoja. Entrevistado el 27 de febrero de 2016 en Jerez de la Frontera.

- José Rojo: profesor del conservatorio de música de Jaén perteneciente a la primera promoción de titulados superiores en la especialidad de guitarra flamenca. También ha destacado en la labor de guitarrista flamenco de concierto y como acompañante al cante. Entrevistado el 14 de julio de 2016 en Jaén.

- Gabriel Expósito: profesor del conservatorio superior de música de Córdoba perteneciente a la primera promoción de titulados superiores en la especialidad de guitarra flamenca. Sobresale también por su labor como guitarrista flamenco de concierto y como acompañante al cante y al baile. Entrevistado el 25 de julio de 2016 en Córdoba. 
- Óscar Herrero: distinguido guitarrista flamenco en su función concertista y en la edición de numerosos métodos didácticos de guitarra flamenca. Entrevista escrita recibida el 5 de octubre de 2016.

- Manolo Sanlúcar: prestigioso guitarrista flamenco considerado uno de los revolucionarios de la guitarra flamenca del siglo XX junto a Paco de Lucía y Serranito. Testimonios recogidos entre el 7 y el 10 de julio de 2017 en Córdoba.

- Marcel Ege: productor musical de Encuentro (Productora suiza pionera en la edición de una colección de vídeos didácticos sobre la guitarra flamenca al inicio de la década de los noventa del pasado siglo). Entrevistado el 9 de julio de 2017 en Córdoba.

- David Pino: profesor del conservatorio superior de Córdoba y cantaor flamenco con un prestigio reconocido en el panorama actual del flamenco. Entrevistado el 9 de julio de 2017 en Córdoba.

- Gerardo Núñez: prestigioso concertista de guitarra flamenca considerado uno de los revolucionarios de la guitarra flamenca del siglo XXI. Entrevistado el 11 de julio de 2017 en Sanlúcar de Barrameda.

- Javier Latorre: prestigioso bailaor, bailarín y coreógrafo. Entrevistado el 11 de agosto de 2017 en Córdoba.

\section{Resultados: procedimientos anejos a la transmisión oral de la guitarra flamenca}

A partir de los datos obtenidos en las entrevistas realizadas, hemos individuado cuatro procedimientos que siempre parecen haber formado parte de la metodología tradicional de enseñanza de la guitarra flamenca (Calahorro, 2018), de forma que entendemos que tanto docente como discente deben tenerlos presentes en el proceso de enseñanza-aprendizaje $\mathrm{y}$, en definitiva, en la formación del guitarrista flamenco.

\subsection{Aprendizaje por imitación}

En la raíz del proceso de enseñanza-aprendizaje de la guitarra flamenca encontramos un método muy particular de transmisión oral, que, en esencia, no es otro que la imitación de un fragmento musical. Este método, referido por Batista (1985) como un aprendizaje bis a bis, es consecuencia en parte de que "quienes tocan la guitarra por lo flamenco, no conocen la música” (Marín, 1902, p. 67). Así recuerda Manolo Franco este proceso en los inicios de su formación, mi maestro por transmisión oral me ponía las falsetas como vehículo para los distintos toques, y además "él ahí, y yo aqui"” (Entrevista a Manolo Franco_4.1.12, p. 336).

Por su parte, Paco Serrano comenta: ese procedimiento de tú a tú [...] desarrolla una serie de cuestiones como la vista, el oido... ese cauce que se produce con el contacto directo de guitarra a guitarra (Entrevista a Paco Serrano_11.11.11, p. 326). Todo este proceso se lleva a cabo de forma oral, como el formato de las entrevistas (Entrevista a José Piñana_22.12.11, p. 331), según señala José Piñana.

Es por tanto una parte fundamental del proceso de enseñanza-aprendizaje de la guitarra flamenca: el profesor propone unas falsetas, variaciones y obras que el alumno va asimilando y afianzando bajo la supervisión del docente. Éste a su vez aconseja sobre detalles técnicos, digitaciones más idóneas, detalles referentes a la 
expresión, etc. La información, como señalaba Sanlúcar en nuestra entrevista, se obtiene a través de la vista y el oído, puestos al servicio de la imitación del pasaje musical propuesto. En el argot de los flamencos, este proceso se identifica con los siguientes verbos: "coger" o "poner" una falseta, una variación o una llamada.

La particularidad de este proceso es que en la guitarra flamenca se ha realizado de forma ágrafa, sin soporte alguno de partitura o de tablatura (Cruces, 2002). Y esto no solo se debe al desconocimiento de la tradición escrita que ha caracterizado a muchos de los flamencos, sino también a que existe un ingente corpus de recursos tradicionales de la guitarra flamenca (variaciones, respuestas al cante o falsetas) que, o bien no están transcritas, o de estarlo, su fidelidad es de dudosa credibilidad.

Pero además, y aunque se esté en posesión del conocimiento necesario para la lectura y transcripción del lenguaje musical, nosotros seguimos defendiendo este proceso sin el soporte escrito. El alumno se somete a una "presión" simbólica que es positiva, porque exige establecer un diálogo con el profesor que le obliga a asimilar los pasajes musicales propuestos en el mismo momento en que tiene lugar el proceso de enseñanza-aprendizaje.

Necesariamente el alumno debe ser un sujeto activo de un proceso en el que su atención es precisamente la base de la asimilación de los diferentes fragmentos musicales. Como apuntaba el catedrático de guitarra Paco Serrano, se desarrollan así en el alumno unas capacidades y habilidades que forman parte de la propia naturaleza de la guitarra flamenca, que tiene mucho de reacción espontánea ante los estímulos externos.

$Y$ es que, se ha de tener en cuenta que en la propia naturaleza de este instrumento no está la memorización de obras de concierto con una estructura cerrada, sino que se estudian pequeños fragmentos (conocidos como falsetas, variaciones o llamadas) aplicados a diferentes contextos musicales (Calahorro, 2018). En todas estas secciones encontramos ejemplos de patrones o fórmulas intercambiables que, de forma improvisada, van enlazándose para formar el discurso musical (Barea, 2016). En estos enlaces debe haber una sólida y fundamentada cohesión armónica, melódica, rítmica y formal. Viene muy a colación aquí el término "tocar", que, en el argot flamenco, se asocia a los diferentes estilos musicales: tocar por bulerías, tocar por alegrías o tocar por tarantas (Castro, 2011). En esencia describe el procedimiento en el que el guitarrista flamenco, acudiendo a ese repertorio personal de secciones musicales (falsetas, variaciones y/o llamadas) de un determinado estilo flamenco para crear su discurso musical, focaliza también su atención en la práctica del acompañamiento, tanto del cante como del baile. Y en ese acompañamiento, el guitarrista ha de mantener el compás, dar respuestas al cante o al baile y armonizarlos (Torres, 2014).

\subsection{Vivencia del acompañamiento al cante y al baile}

El análisis y estudio realizado de los tratados y métodos de guitarra barroca, guitarra clásico-romántica - precedentes musicales de nuestra música flamenca-y de guitarra flamenca, han arrojado información de gran valor que corrobora y confirma la pervivencia de una transmisión oral de conocimientos en la que la ocasión interpretativa del acompañamiento vocal y de danzas ha tenido un lugar privilegiado (Calahorro, 2018). Este hecho era ratificado también por todos los profesores de la provincia de Córdoba de la especialidad de guitarra flamenca que formaron parte de la investigación de Alicia González: además de formarse en el conservatorio, admitieron que se habían beneficiado del ambiente de las peñas flamencas y de las academias, es decir, 
de lo que en el argot flamenco se conoce como "formarse en la calle" (González, 2016, p. 323).

Paco Cepero contaba que le pidieron un consejo sobre qué debía hacer un famoso guitarrista que había ganado con 14 años el famoso Premio Nacional de Córdoba en la modalidad de guitarra de concierto: Le dije: 500 bautizos, 600 bodas y que se rompa mucho las uñas. Y que se vaya a un tablao (Entrevista a Paco Cepero_27.2.16, p. 345). Con esta declaración deja claro que, aunque hayan aflorado las aptitudes necesarias para ser un gran concertista, no hay duda de que la vivencia favorecida por la propia experiencia del acompañamiento al cante y al baile es fundamental para dotar de fundamento y solidez el proceso de enseñanza y aprendizaje de la guitarra flamenca.

El guitarrista debe saber acompañar un mismo estilo cantado por diferentes cantaores, pues, en muchos de los casos, habrá ligeras modificaciones en la expresión, la dinámica o el tempo. David Pino, profesor de cante flamenco del conservatorio superior de Córdoba, nos comentaba que el alumnado de conservatorio tan solo escucha al profesor de cante del conservatorio, pero que lo ideal sería que pudieran escuchar y acompañar a muchos más. Y eso, como él mismo reconocía, te lo da la vivencia paralela. También nos lo aseguraba Juan Manuel Cañizares: la calle, la vivencia, en definitiva, la experiencia directa es el pilar fundamental para vivir el flamenco (Entrevista a Juan Manuel Cañizares_13.1.12, p. 341). Conectando con la anécdota referida más arriba de Paco Cepero en relación a los 500 bautizos y 600 bodas por las que debía pasar un guitarrista que muy joven consiguió un primer premio como concertista, Paco Serrano nos decía que para él la vivencia del acompañamiento fue fundamental y que uno se curte en mil batallas, y si no las pasas, no te curtes. [...] Esa ha sido la base de mi formación, al margen de mis maestros: el haber estado acompañando con 15 años a cantaores (EPS_11.11.11, p. 327). También lo asevera el guitarrista Manolo Franco: Todos los grandes (guitarristas) han pasado por tocar para bailar, tocar para cantar, y te da esa "cosa" que después un guitarrista flamenco es: la composición (EMF_4.1.12, p. 337). Y si hiciéramos un rastreo de los grandes concertistas de la historia de la guitarra flamenca, todos probablemente admitirían haber comenzado su andadura en el flamenco por medio del acompañamiento. El mismo Sanlúcar nos reconocía en la entrevista que con trece años ya estaba acompañando a Pepe Marchena (Entrevista a Manolo Sanlúcar_7.7.17, p. 374).

En general, creemos no exagerar al afirmar que todos los guitarristas entrevistados (Paco Serrano, José Piñana, Manolo Franco, Juan Manuel Cañizares, Paco Cepero, José Rojo, Gabriel Expósito, Óscar Herrero, Manolo Sanlúcar y Gerardo Núñez) admiten que en el inicio del aprendizaje de la guitarra flamenca debe haber una sólida presencia de la labor acompañante del cante (Calahorro, 2018).

Es significativa en este sentido la experiencia vivida en el Conservatorio Superior de Córdoba de dos alumnos Erasmus del Conservatorio de Rótterdam. Según nos contó Paco Serrano:

Los alumnos Erasmus están dando acompañamiento al cante y al baile con Manolo Franco y el Niño de Pura. Complementan esto con la participación en las actividades de las peñas, y están encantados, y me confiesan que encuentran ahi la base de su formación. [...] Lógicamente esa práctica en directo que desarrolla facilidades de acompañar a cualquiera en cualquier momento, de tener que 
responder a un cantaor si no sale a tono, a compás...y sin embargo no te puedes parar, tienes que hacer que aquello suene bien (EPS_11.11.11, p. 327).

En efecto, un guitarrista flamenco debe saber acompañar a un cantaor que desafina o que no respeta el compás. Cuando comienza la interpretación del cantaor, instantáneamente el guitarrista ha de afinar el oído y saber en qué tonalidad está cantando. Mediante ese acompañamiento se irán despertando y desarrollando las facultades propias de la guitarra flamenca como son:

- Capacidad de respuesta ante los tercios cantados.

- Diferenciación de los tonos por los que va pasando el cantaor para así aplicar la correspondiente respuesta armónica y acordes de paso.

- Adaptación al tempo de cada cantaor, sabiendo jugar con la elasticidad propia del compás en cada uno de los estilos musicales del flamenco (Berlanga, 2014).

- Conocimiento de los estilos existentes dentro de cada palo para poder aplicar el carácter y matiz deseado.

- Utilización de ese repertorio tan característico de recursos musicales, falsetas, llamadas, cierres y variaciones que los guitarristas utilizan de forma intuitiva y espontánea en la dinámica propia de este acompañamiento, sin que ello suponga un obstáculo para poder acompañar.

Este proceso debe darse de forma similar con respecto al baile, pues aunque en la actualidad la puesta en escena del baile responde más a un esquema fijo concienzudamente montado entre los músicos y el cuerpo de baile, también existen partes cuya interpretación depende del momento, apareciendo fragmentos improvisados, con diferentes velocidades, duraciones y/o figuraciones rítmicas (Brao, 2014). La propia adaptación al ritmo, expresividad e intencionalidad del bailaor ha de ser captada de manera inmediata por el guitarrista flamenco. En estos montajes siempre surgen nuevas ideas musicales, lo que también fomenta la creatividad e improvisación o la asimilación instantánea de las figuraciones rítmicas del baile. Como comentaba Javier Latorre, es un intercambio permanente en el que el guitarrista ha de ser capaz de compartir lo que él sabe con el resto y recibir abiertamente las aportaciones de los demás.

En definitiva, en la base de la formación del guitarrista flamenco debe haber una presencia fundamental de la labor acompañante, tanto del cante como del baile. No se puede concebir de otra forma. Además, ese acompañamiento ha de desarrollarse en diversos entornos (peñas, tablaos o academias de baile) y con diferentes artistas, potenciándose esa capacidad camaleónica del guitarrista, capaz de adaptarse a la idiosincrasia de cada cantaor $\mathrm{y} / \mathrm{o}$ bailaor.

\subsection{Empleo del oído para la adquisición de recursos y obras}

Hablando de las capacidades que caracterizaban al guitarrista flamenco, José Rojo decía que los guitarristas flamencos siempre han tenido esa intuición musical, porque el oído era el principal órgano de recepción (Entrevista a José Rojo_14.7.16, p. 352). En efecto, en el proceso al que aquí aludimos no interviene la vista, sino que es el oído el único medio receptor que permite copiar literalmente lo que estamos escuchando para posteriormente interpretarlo con el instrumento. Así definía este proceso 
Paco Serrano: escuchando los discos e imitándolos, delante de un tocadiscos, por imitación (EPS_11.11.11, p. 328). Este procedimiento, también aludido por Expósito, exige del músico la capacidad de focalizar la atención sobre diferentes variables de ese fragmento musical como la melodía principal, la armonía acompañante, los golpes en madera o las secuencias rítmicas.

Debe tenerse en cuenta que mediante este proceso de audición no solo se desarrolla la destreza de captar información, sino también la de filtrar la información irrelevante. La escucha óptima, por tanto, se refiere a la habilidad de usar el oído focalizando conscientemente la atención en un sonido de entre los demás sonidos del ambiente. Son varias las habilidades que proporciona al músico el uso de este modo de aprendizaje:

- Permite trabajar el oído relativo, gracias a la gran precisión requerida para discernir las diferentes notas que se nos presentan. El oído relativo consiste en la capacidad para percibir relaciones melódicas con independencia de la ubicación real. Actúa de forma más elaborada (no es automático) que el absoluto, pues debe orientarse a partir de puntos de referencia tonales, interválicos, etc. La importancia para el guitarrista flamenco del desarrollo de esta habilidad, reside en que para la labor de acompañamiento al cante es de vital importancia la diferenciación de los tonos por los que el cantaor va pasando con el fin de ir adaptándose y glosar armónicamente a éste. Además, debido al uso de la cejilla para acompañar al cante, es importante el desarrollo de un oído relativo, donde los tonos que ponemos para acompañar no son realmente los que suenan, pues se trabaja por dibujos, posturas y acordes, de ahí su relatividad.

- Potencia la búsqueda de digitaciones más ergonómicas para el guitarrista flamenco, ya que, debido a la variedad de posibilidades para realizar un fragmento, el guitarrista buscará siempre la más cómoda y eficaz. En este sentido cobran significado pleno las palabras de Piñana, pues cuando uno se propone interpretar lo que está escuchando tú estás buscando y te vas a otra cosa, y junto con esa inquietud de composición y de creación vas conformando tu propia personalidad (EJP_22.12.11, p. 332). En este proceso aparecen nuevas posiciones, acordes, o melodías que resultan sorpresivas y que muchas veces sirven de motivación para la creatividad personal.

Sin embargo, apuntamos que con la proliferación de las nuevas tecnologías, y en concreto, internet y medios audiovisuales donde el material musical se detalla minuciosamente, este proceso puede quedar relegado a un segundo plano, pues el tiempo empleado para obtener música haciendo uso solo del oído es mucho mayor que si podemos utilizar el formato audiovisual, donde sí visualizaríamos las posiciones y técnicas empleadas.

\subsection{Composición guitarrística}

El estudio de la guitarra flamenca ha de comenzar por la interpretación del repertorio tradicional de falsetas, variaciones y obras de los diferentes estilos flamencos. El decantarse por un guitarrista u otro dependerá del profesor que imparta esas clases $\mathrm{y}$, posteriormente, del gusto musical de cada guitarrista. Sin embargo, a diferencia de otros instrumentos donde la interpretación suele perdurar durante toda la carrera artística, Manolo Franco nos decía que la lógica imperante entre los guitarristas 
flamencos es que ese aprendizaje, sobre la base de una dilatada experiencia acompañando al cante y al baile, se aboque a la adquisición de un repertorio original de composición propia. Así nos lo reconocía Gerardo Núñez:

En el cante se ha premiado la interpretación y en la guitarra no. Cuando tocas algo de Paco de Lucía decían que lo que tocabas era de él. Sin embargo cuando alguien hace la malagueña de Chacón, todo el mundo:-olee...

No han aplaudido la creación [refiriéndose al cante] y los guitarristas nos hemos llevado la peor parte y hemos tenido que desarrollar todo a la vez. Hemos tenido que trabajar el doble. Y por eso la guitarra ha avanzado muchísimo: si en la guitarra se premiase la interpretación estaríamos tocando todavía las "cosas" de Sabicas y de Montoya (Entrevista a Gerardo Núñez_11.7.17, p. 389).

En efecto: una innovación en el cante flamenco no tendrá la misma acogida que en la guitarra flamenca, pues en el cante siempre ha existido un cierto recelo a la variación de los estilos considerados matrices o canónicos. Respecto a esa tendencia de prosperar y adquirir una personalidad en el toque, resultan significativas las palabras de Paco Cepero:

Después de mirarme en el espejo de los que a mi me gustaban: Melchor de Marchena, Manolo de Huelva, Diego del Gastor, Montoya... Lo que yo he luchado toda mi vida era por no parecerme a nadie. Lo que tienes que hacer es no escuchar. Yo tengo discos ahi que me los regalan pero no los escucho porque puedo tener la tentación (EPC_27.2.16, p. 346).

A este respecto, aportamos aquí la visión de Scionti, quien define muy bien ese proceso de nueva aportación mientras hay un respeto de esa tradición:

La modificación de la tradición incluye la capacidad de asimilar algo considerado ajeno a la tradición, la cual se puede interpretar como la incorporación de ideas o prácticas nuevas y por tanto la evolución de una tradición, eso sí, todo filtrado y condicionado por las normas fundamentales de una tradición y por tanto ejerciendo algo de 'autoridad' sobre las prácticas (2017, p. 192).

En definitiva, una vez que se ha consolidado un determinado toque, la naturaleza del propio guitarrista será la de ir creando sus propias variaciones, falsetas y, como culminación de dicho proceso, obras de concierto. Y en la base de este proceso, Expósito nos verificaba que una dilatada experiencia en la labor acompañante de la guitarra flamenca propiciará y motivará la creatividad y originalidad en la composición personal. Esta característica, como seña de identidad inherente al guitarrista flamenco, ha de seguir perpetuándose.

\section{Conclusiones}

Las entrevistas con informantes competentes y autorizados aportan nuevos argumentos sobre la vigencia del aprendizaje de la guitarra flamenca por imitación entre 
profesor y alumno, fomentándose así una serie de aptitudes que permiten actuar con fluidez y espontaneidad ante estímulos externos Además confirman que esta imitación no puede ir separada de la vertiente clave del acompañamiento al cante y al baile, aprendizaje privilegiado a través del oído. Si la imitación es esencial en sus procesos iniciales, ésta es la que mejor facilita el repertorio musical de falsetas y variaciones para desempeñar la práctica del acompañamiento al cante y al baile. La capacidad de adaptación rítmica y armónica al discurso del cante y el baile han de aflorar muy poco después con la puesta en práctica de lo aprendido durante las sesiones de acompañamiento. En esa práctica conviene conocer todo el repertorio de estilos flamencos a través de la vivencia, interactuando y acompañando a diferentes artistas en entornos no reglados (peñas, tablaos, académicas de baile o reuniones informales). En segunda instancia estará la labor concertista, que ha de ser una ilación de la labor acompañante, requiriendo para su desempeño una dilatada experiencia previa en las dos funciones anteriores: acompañante del cante y del baile.

Paralelo a esta enseñanza, hemos visto que es igualmente importante fomentar que el alumnado investigue autónomamente y utilice su oído para extraer nuevo material musical de diferentes guitarristas, pasando a formar parte de su repertorio. Este último proceso también propicia la creatividad y la innovación mediante la búsqueda de nuevos acordes, escalas o ritmos. Son aptitudes que invitan a la innovación personal dentro del repertorio musical.

El músico flamenco debe desarrollar la faceta de intérprete pero también de creador, pues forma parte de la idiosincrasia del guitarrista flamenco la necesidad de desarrollo de un repertorio personal de falsetas, variaciones y obras de cada estilo flamenco.

Como consecuencia de la institucionalización de la guitarra flamenca estamos plenamente convencidos de que la principal asignatura ha de ser la de acompañamiento, iniciándose por el cante y continuando con el baile. Paralelamente, la metodología de enseñanza de la guitarra flamenca, ya sea dentro de un contexto legislado o fuera de éste, ha de articular el modo en que las cuatro facetas expuestas unas líneas más arriba sean tenidas en cuenta de cara a fomentar en el profesorado una enseñanza por imitación, instando siempre a que el alumno sea partícipe de la vivencia del acompañamiento al cante y al baile y a que desarrolle su oído, fomentándose paralelamente la creatividad y la composición propia.

Bajo un prisma musicológico, esta institucionalización supone una dignificación de la música flamenca, que se equipara a cualquier otra música que también goce de presencia en el conservatorio. Pero esa equiparación no puede traducirse en un trasvase metodológico o actitudinal. La naturaleza y rasgos que forman parte de la idiosincrasia de la música flamenca siempre deberán ser tenidos en cuenta, valorados y transmitidos. Así se conseguirá que la integración entre la tradición y la modernidad sea plena: suplir las antiguas carencias musicales de los guitarristas flamencos sin que vaya en detrimento de sus puntos fuertes.

\section{Referencias bibliográficas}

Amat, J. C. (1596). Guitarra española de cinco órdenes. Barcelona: s.e.

Batista, A. (1985). Manual Flamenco. Madrid: Edición de autor.

Barea, M. A. (2016). La improvisación en guitarra flamenca. Análisis musicológico e implementación didáctica. Tesis doctoral, Universidad de Sevilla, Departamento de 
Didáctica de la Lengua y la Literatura y Filologías Integradas. Barcelona, Joseph Bro. Disponible en https://idus.us.es/xmlui/handle/11441/57175

Berlanga, M. A. (2014). La originalidad musical del flamenco: el compás. Revista Sinfonía Virtual, núm. 26, 2014. Disponible http://www.sinfoniavirtual.com/flamenco/flamenco_ compas.pdf

Bolívar, A. (1995). El conocimiento de la enseñanza. Epistemología de la investigación curricular. Granada: FORCE.

Bouvier, J. C., Bremondy, H.P., Joutard, P., Mathieu, G. y Pelen, J.N. (1980). Tradition orale et identité culturelle. Problèmes et Méthodes. París: C.N.R.S.

Brao, E. (2014). Baile Flamenco. Observación y análisis del Taranto en los ámbitos profesional y académico. Tesis doctoral, Universidad de Murcia, Departamento de la actividad física y del deporte. Disponible en http://tdx.cat/bitstream/handle/10803/294999/TEBM. pdf? sequence $=1$ \&isAllowed $=\mathrm{y}$

Briceño, L. (1626). Metodo mui facilissimo para aprender a tañer la guitarra a lo español. París: Pedro Ballard.

Calahorro, M. A. (2017). La metodología tradicional de enseñanza en los tratados de guitarra flamenca del siglo XX. Música Oral del Sur, n. 14, 103-121. Disponible en http:// www.centrodedocumentacionmusicaldeandalucia.es/opencms/documentacion/revistas/ articulos-mos/la_metodologia_tradicional_de_ensenanza_en_los_tratados_de_guitarra flamenca_del_siglo_xx.html

Calahorro, M. A. (2018). La metodología tradicional de enseñanza y aprendizaje de la guitarra flamenca: un estudio diacrónico y sincrónico. Tesis doctoral, Universidad de Granada, Departamento de Historia y Ciencias de la Música. Disponible en: http:// digibug.ugr.es/handle/10481/52366

Calahorro, M. A. (2019). La legislación en torno a la guitarra flamenca: recorrido diacrónico y propuesta de mejora con base en su naturaleza popular. Telethusa (Revista de Investigación de Flamenco) 12, 5-17. Disponible en http://www.flamencoinvestigacion. es/articulos-1214-01-2019-guitarra/

Cano, M. (1986). La guitarra. Historia, estudios y aportaciones al Arte Flamenco. Córdoba: Universidad de Córdoba y Monte de Piedad y Caja de Ahorros de Córdoba.

Castro, G. (2011). Origen del "tono" y "toque de taranta". Revista de Investigación sobre flamenco La madrugá, núm. 5., 109-116. Disponible en https://revistas.um.es/flamenco/ article/view/139871

Cera, M. (2006). En torno a la guitarra flamenca y la notación musical. Revista Musicalia, $n^{\circ} 4,111-133$.

Cervantes, M. (1613). Novelas exemplares. Madrid: Iuan de la Cuesta. Disponible en http:// bdh-rd.bne.es/viewer.vm?id=0000114398\&page $=1$

Corbetta, P. (2003). Metodología y técnicas de investigación social. Madrid: McGraw Hill.

Cruces, C. (2002). Una primera aproximación a las metodologías de estudio del flamenco. Perspectivas, necesidades y líneas de trabajo. Patrimonio Musical, 63-107.

Delgado, L. (1906). Método de Guitarra en Serio y flamenco para aprender a tocar sin necesidad de Maestro. Palencia: s.e. Disponible en http://bdh.bne.es/bnesearch/CompleteSearch.do?f ield $=$ todos \& text $=$ lucio + delgado $\&$ showYearItems $=\&$ exact $=$ on \& textH $=\&$ advanced $=$ false $\&$ completeText $=$ \&pageSize $=1$ \&pageSizeAbrv=30\&pageNumber $=2$

Dembowski, C. (1841). Dos años en España durante la guerra civil, 1838-1840. París: Charles Gosselin.

Estébanez, S. (1847). Escenas andaluzas. Madrid: Imprenta de Don Baltasar González. Disponible en http://bdh.bne.es/bnesearch/CompleteSearch.do?field=todos\&text=escen 
as + andaluzas $\&$ show YearItems $=$ \&exact $=$ on \& text $H=\&$ advanced $=$ false $\&$ completeText $=\&$ pageSize $=1 \&$ pageSizeAbrv $=30$ \&pageNumber $=11$

Ford, R. (1845). A hand-book for travellers in Spain, and readers at home. Londres: J. Murray. González, A. (2016). Un estudio descriptivo de la enseñanza del flamenco: las percepciones del profesorado de guitarra flamenca en la provincia de Córdoba. Tesis doctoral, Universidad de Granada, Departamento de Didáctica y Organización Escolar.

Grecos, J. (1973). La Guitarra flamenca. Madrid: Unión Musical Ediciones.

Jorge, M. (1860). Modo práctico para aprender el rasgueo de guitarra. Madrid: s.e. Disponible en http://bdh.bne.es/bnesearch/CompleteSearch.do?visor=\&text=\&field1val $=\% 22$ Jorge + Rubio $\% 2 \mathrm{c}+$ Mat $\% \mathrm{c} 3 \%$ adas $+\mathrm{de} \% 22 \&$ show YearItems $=\&$ field $1 \mathrm{Op}=\mathrm{AND} \& \mathrm{n}$ umfields $=1 \&$ exact $=$ on \& text $\mathrm{H}=\&$ advanced $=$ true $\&$ field $1=$ autor \&completeText=\&pageSi $\mathrm{ze}=1 \&$ pageSizeAbrv $=30$ \&pageNumber $=9$

Joutard, P. (1983). Ces voix qui nous viennent du passé. París: Hachette. [Trad. Castellana. Esas voces que nos llegan del pasado, México: FCE, 1986]

Ley Orgánica 1/1990, de 3 de octubre, de Ordenación General del Sistema Educativo. BOE, 4 de octubre de 1990, núm. 283. pp. 28927-28942. Disponible en https://www.boe.es/ buscar/doc.php?id=BOE-A-1990-24172

Ley Orgánica 2/2006, de 3 de mayo, de Educación. BOE, 4 de mayo de 2006, núm. 106. pp. 17158-17207. Disponible en https:/www.boe.es/diario_boe/txt.php?id=BOE-A-2006-7899

Marín, R. (1902). Método de Guitarra. Por Música y Cifra, Aires Andaluces. Madrid: Dionisio Álvarez.

Ong, W. (2006). Oralidad y escritura: Tecnología de la palabra. Buenos Aires: Fondo Cultura Económica Argentina. [1 ${ }^{\mathrm{a}}$ ed.: Londres, 1982]

Pacheco, C. (2003). El flamenco: una visión educativa y cultural. Revista Musicalia, $\mathrm{n}^{\circ} 1$.

Peñalba, M. (1877). Cuaderno de guitarra y bandurria por cifra por el sistema moderno. Logroño: Imprenta de Federico Sanz. Disponible en http://bdh-rd.bne.es/viewer. vm?id=0000129042\&page $=1$

Pérez, D. (2005). Paco de Lucía. La evolución del flamenco a través de sus rumbas. Cádiz: Servicio de Publicaciones de la Universidad de Cádiz.

Scionti, I. (2017). La guitarra flamenca: tradición y renovación. Tesis doctoral, Universidad de Sevilla: Departamento de Antropología Social.

Torres, N. (2009). De lo popular a lo flamenco: aspectos musicológicos y culturales de la guitarra flamenca (siglos XVI-XIX). Tesis doctoral, Universidad de Almería.

Torres, N. (2011). El estilo "rasgueado" de la guitarra barroca y su influencia en la guitarra flamenca: fuentes escritas y musicales, siglo XVII. Revista Música oral del Sur, 9, 288320. Disponible en http://www.centrodedocumentacionmusicaldeandalucia.es/opencms/ documentacion/revistas/articulos-mos/el-estilo-rasgueado-de-la-guitarra-barroca-y-suinfluencia-en-la-guitarra-flamenca-fuentes-escritas-y-musicales-siglo-xvii.html

Torres, N. (2014). Claves para una lectura musical de la obra de Paco de Lucía. Revista de Investigación sobre flamenco La madrugá, núm. 11, 1-61. Disponible en http://revistas. um.es/flamenco/article/view/221811/172781

Vargas y Guzmán, J.A. (1773). Explicación de la guitarra. Cádiz: s.e.

Winchester, H. (1996). Ethical Issues in Interviewing as a Research Method in Human Geography. Australian Geographer, vol. 2, Nº1, 117-131. 